1 Type of the Paper (Article, Review, Communication, etc.)

2 Image Judgment Auxiliary System for Table Tennis

3 Umpiring Under Low Light Conditions

4

\author{
Chang-Hung Hung \\ Office of Physical Education, National Chin-Yi University of Technology, Taichung 41170, Taiwan \\ * Correspondence: hongjh@ncut.edu.tw; Tel.: +886-4-23924505
}

\begin{abstract}
In table tennis competitions the rule violation judgment with the greatest controversy is the height of the ball serve. This is because inaccuracy in the ball height judgment, which results in erroneous judgment, is unavoidable. Thus, we designed an automatic image judgment auxiliary system for table tennis ball height during service in this study. We used a high-speed camera to record the ball toss in the table tennis service. The designed algorithm architecture can automatically search for the ball and the position of the hand action under low light source conditions. The algorithm is mainly divided into hue-saturation-value color space processing and morphology processing using Hough transform to search for the circular ball. Experiment result shows that color segmentation can successfully and accurately determine the ball position under low light conditions. The morphology method can find the position of the hand and help determine the moment when the ball leaves the hand during the service ball toss. Finally, the actual size of the target is used to estimate the actual distance unit represented by the image pixel.
\end{abstract}

Keywords: table tennis, hue-saturation-value (HSV) image segmentation, low light source, automatic tracking

\title{
1. Foreword
}

Table tennis is currently a popular competition sport. To achieve good performance, the athlete must continuously pursue improvements in table tennis skills. Thus, the setting of the rules and requirement for athletes to comply with competition rules is becoming stricter. The judgment of rule violation in all types of sports competitions almost always rely on the professionalism and subjective judgment of the umpire. However, even though the umpires have gone through professional training to make their judgment more objective, uncertainties in the umpire's subjective judgment and level of physical concentration can occasionally cause erroneous competition judgment. These erroneous judgments can cause significant negative impact on the athlete's psychological status, thereby, affecting the competition results. In table tennis competitions the rule violation that currently has the greatest controversy is the height of the serve ball. A table tennis service usually takes a few seconds to complete. However, there are over ten observations an umpire needs to take and make a judgment before or just after the service is completed. This is a very complex task and requires a lot of judgments, even for an experienced umpire [1]. According to Article 2.6.2 of the International Table Tennis Federation (ITTF) Handbook 2017 "The server shall then project the ball near vertically upwards, without imparting spin, so that it rises at least $16 \mathrm{~cm}$ after leaving the palm of the free hand and then falls without touching anything before being struck" [2]. Different than out of bounds call, where there is a clear boundary as a reference, there is no clear reference for the minimum $16 \mathrm{~cm}$ height in a table tennis service, and it is difficult for umpires to accurately judge whether a violation has occurred with their bare eyes. The inaccuracy of the ball height judgment has resulted in protest 
from the athletes regarding this penalty. Thus, the use of image technology to help umpires with their judgment is necessary.

Many sports have already introduced image technology to help umpires make the final judgment or to propose ruling change for erroneous judgments. Some examples are the Hawk-Eye in tennis and badminton and the goal-line technology and VAR in soccer. These auxiliary judgment systems can provide clear image proof for judgment that has been questioned. Currently, the judgment in table tennis umpiring mostly relies on the umpire's experience and on-site intuitive response. Image technology has not yet been applied to determine the accuracy of the umpire's judgment. Thus, the competitors do not have a way to challenge questionable judgment. Thus, researchers have begun to think about how to introduce image technology to help with umpire judgments in table tennis competitions. Wong believes the employment of videography, image processing and artificial intelligence (AI) technologies can help evaluate table tennis service [3]. Myint et al. proposed an algorithm to track table tennis in the hope of creating an automatic umpiring system [4]. Table tennis umpiring auxiliary system is still in the developmental stage. Therefore, we focused on the building of a table tennis umpiring auxiliary system. Desai et al. presented an algorithm for detecting and tracking ping pong balls in sports videos. The proposed method uses motion as the primary cue for detection. The detected object is tracked using the multiple filter bank approach [5]. Wong used videography, image processing and artificial neural network (ANN) technology to help determine the table tennis serving height [1]. Wong continued to develop an intelligent system which is able to identify and track the location of the ball from live video images and evaluate the service according to the service rules [3]. Wong and Dooley presented a system to automatically detect and track the ball during table-tennis services from real match videos [6]. The first task in calculating the height of the serve ball in table tennis is identifying the circular table tennis ball and tracking its movement. Wong used ANN technology to detect the circular ball. The disadvantage is that the ANN model requires training to be able to make the detection. Thus, the ANN model can be easily affected by background interference and produce detection error. Wong then used multi-layer perceptron (MLP) and the radial basis function network (RBF) in improve table tennis identification capability. However, this method is only suitable for analyzing one picture [3]. The advantage of using circular Hough transform to find circular objects is that this transform can resist noise and the algorithm is robust [7] - [9] [18]. For the image noise filter, not only is the commonly seen averaging filter and median filters used, but morphology based processing method can also be used to filter out the noise. This includes erosion, dilation, opening, and closing [10][11]. The most important key is the color separation, which separates the background and the foreground. Using color separation technology to separate the background and foreground of the target can significantly reduce calculation quantity. The first difficulty that this technology will encounter is the lighting problem [12][13]. Lighting stability and lighting strength both affect the color separation status. The selected color model can also affect the effectiveness of the separation. Many skin color detections related studies have mentioned this problem. Chaves-González et al. compared red-greenblue (RGB), cyan-magenta-yellow (CMY), $\mathrm{Y}$ is the luminance component and UV stand for two chrominance components (YUV), luminance $(\mathrm{Y})$ in-phase quadrature $(\mathrm{YIQ})$, green $(\mathrm{Y})$-blue $(\mathrm{Pb})$-red $(\mathrm{Pr})(\mathrm{YPbPr}), \mathrm{Y}^{\prime}$ is the luma component and $\mathrm{CB}$ and $\mathrm{CR}$ are the blue-difference and red-difference chroma components ( $\mathrm{YCbCr}$ ), luminance-chroma-blue-chroma-red $(\mathrm{YCgCr})$, the colour space used in the SÉCAM analog terrestrial colour television broadcasting standard (YDbDr), hue-saturationvalue (HSV) , hue-intensity-saturation (HIS), and 1931 international commission on illumination XYZ color space (CIE-XYZ) color models. Among these, the HSV is the most suitable for detecting skin color [14][17]. Cho et al. proposed using adaptive threshold to detect skin in HSV color space [15]. Sigal et al. also built a skin color model and used this model in HSV color space [16]. The key in image technology is the architecture design of the image processing algorithm. Thus, we separated and transformed HSV color in this study and used Hough transform and morphology calculation to build an automatic image processing procedure for table tennis service in low light conditions. 


\section{Research Method}

The research architecture of this study is described in section 2.2. Section 2.1 describes the image collection, section 2.2 describes the color image processing (including the automatic sphere tracking and hand position determination), and section 2.3 describes the transformation relationship between image pixel and real size. The objective of this study is to determine the location of the ball and the hand's upper edge, which are used to determine the ball height during the serve in relation to the hand. This relation is used to determine the legality of the height of the serve ball, which can provide a reference for researchers who are building an auxiliary judgment system.

\subsection{Image collection}

In this experiment we used an ix-cameras i-SPEED 210 (F-mount $1280 \times 1024$ Resolution, $2 \mu \mathrm{s}$ shutter, $79500 \mathrm{fps}$ ) high-speed camera. The camera is placed in a position parallel to the table's end line. The shooting distance is $200 \mathrm{~cm}$ away from the table's side line. The camera is at $100 \mathrm{~cm}$ vertical height away from the ground. The high-speed camera is used to extract the ball toss action during table tennis services. Sampling takes 525 frames per second and the image size is $880 \times 1194$ pixels. Image processing is conducted on each frame during the post-analysis. Generally, high-speed camera recording requires sufficient light. However, shinning an excessively strong light source on the server not only affects the server's vision, but a does not conform to the environmental light source at an actual competition. Filming should not allow strong light to affect the server's vision. Therefore, lack of strong light shining on the server conform more to actual competition lighting. Thus, the highspeed shooting in this study was done with a low light source. The ball used was of a type designated for the 2016 Rio Olympics.

\subsection{Color image processing procedure}

Serving in table tennis can be divided into serving preparation action, the instant that the ball leaves the hand, the apex of the ball toss, and when the ball starts to fall. The objective of this study is to obtain the image track from when the ball leaves the hand to the apex of the ball toss, and then calculating the ball height. Algorithm processing architecture is shown in Figure 1. The challenge to the auxiliary judgment system is that it must make a judgment within a few seconds after the serve is complete. Therefore, the region of interest (ROI) must be cut out from each frame to reduce information size and remove unnecessary noise, which increases the calculation and determination speed. Type 1 and Type 2 image processing are then used to find the ball and hand's approximate position. HSV color space based Type 1 and Type 2 separates the ball and hand's approximate position. The difference between the two image processing methods is that they have different threshold parameters. The partial screenshot of the frame is as shown in Figures 2 and 3. 


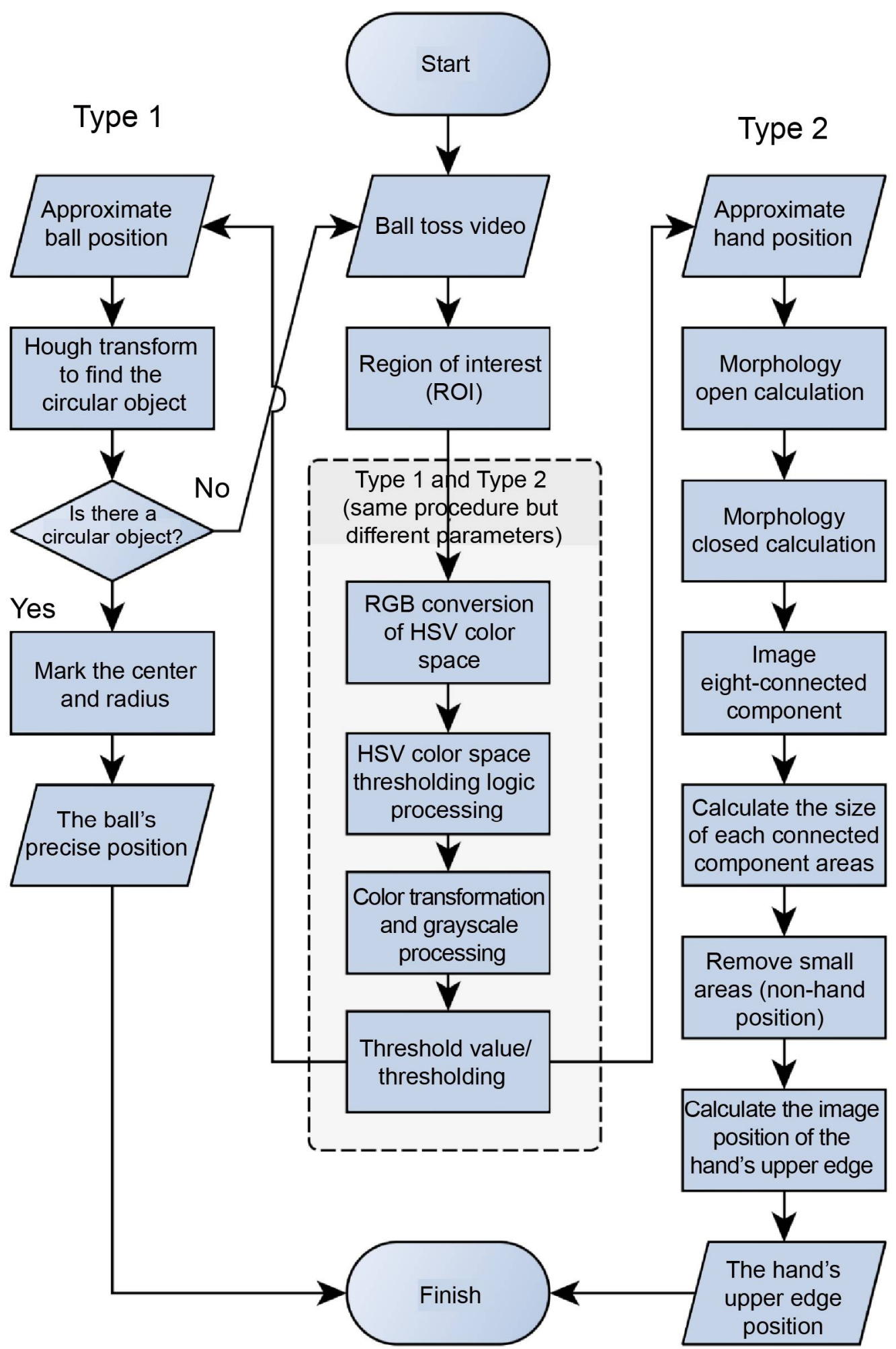

132 Figure 1. Automatic image processing architecture 

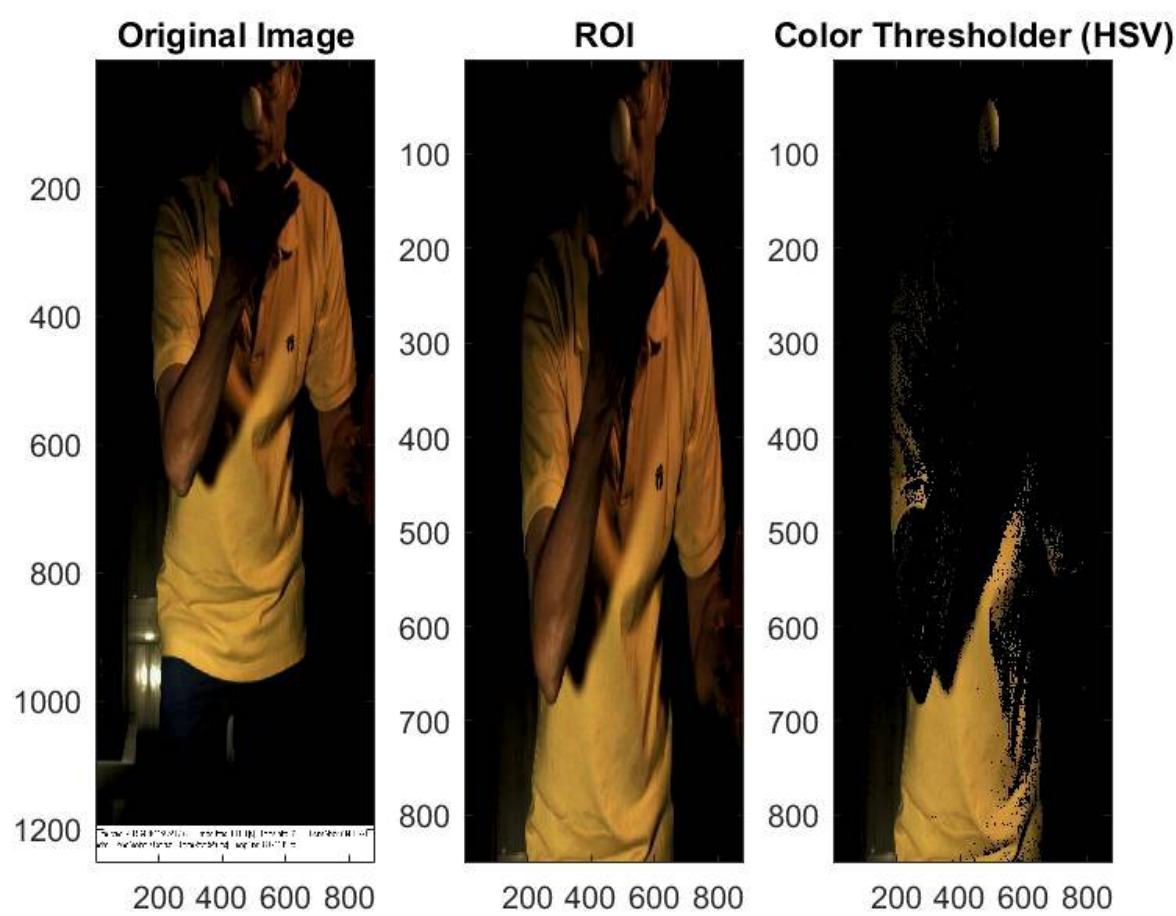

Figure 2. ROI and Type 1 single frame result

135 136

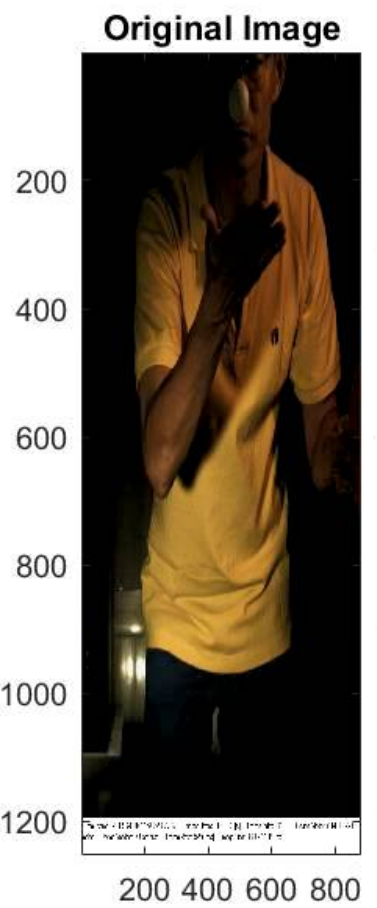
figure)
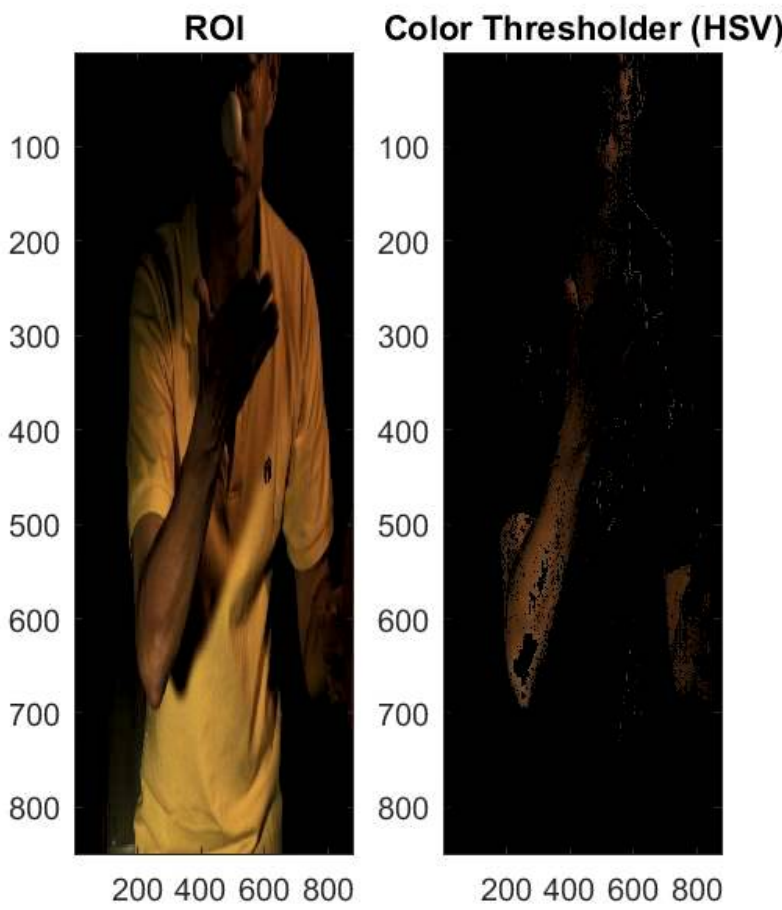

Original image (left figure), ROI image (middle figure), and HSV color segmentation result (right

Figure 3. ROI and Type 2 single frame result 
The approximate ball position can already be found in the image after Type 1 processing. To find the precise position, image thresholding was first conducted and then Hough transform was used to find the circular object and determine whether the circular object has appeared. If the circular object appears, this means that the ball has left the palm of the server. If the circular object appears, then the center and radius information is recorded. This information is the ball's precise position. The processed partial frame is as shown in Figure 4.
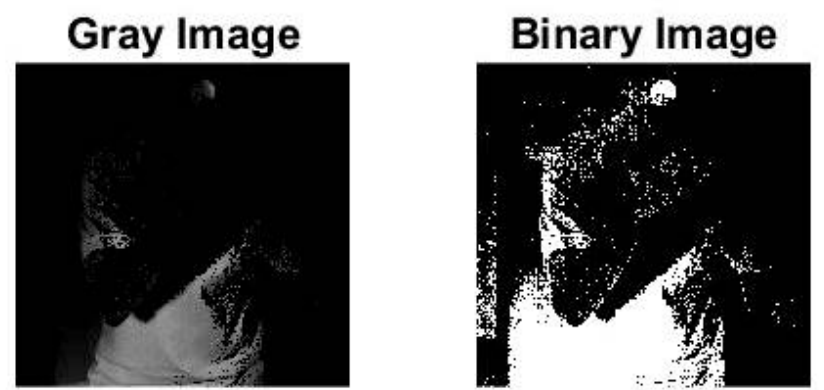

\section{Circular Hough Transform}

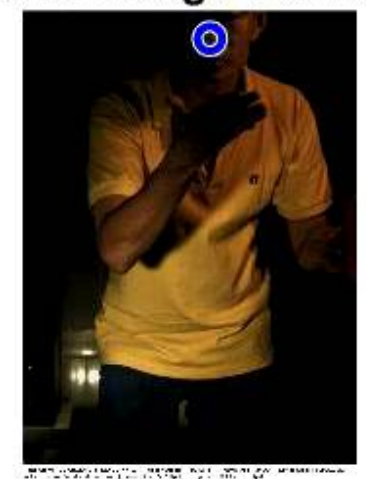

Figure 4. Circular object search result with grayscale, thresholding, and Hough transform

The approximate hand position can be found in the image after Type 2 processing. To find a more accurate position, we first used morphology to conduct opening and then closing to solve the noise problem. Afterwards, image connected component analysis was used find the upper edge of the hand. Methods used included calculating the eight-connected components and the size of the connected components. The result was used to eliminate images that do not include the hand position. Finally, thresholding of the image's upper edge was used as a basis for the hand's upper edge. The processed partial frame is as shown in Figure 5.
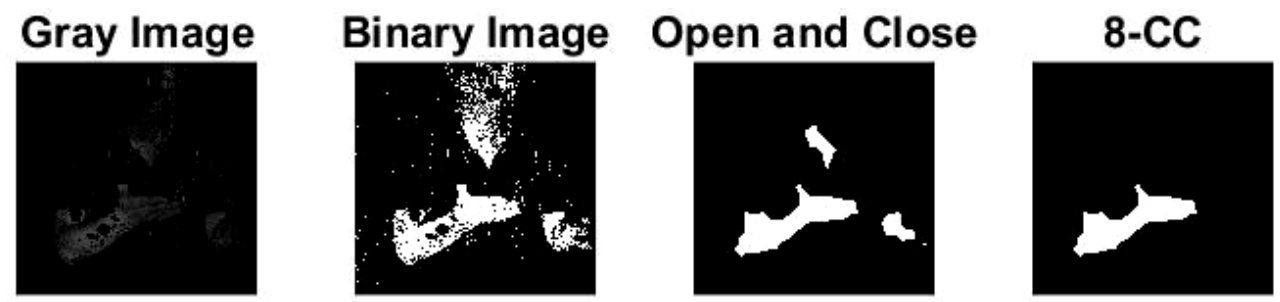

Figure 5. Image processing method for finding the hand position

\subsection{Conversion relationship between the image pixels and actual size}

In this study we used the table's white end line to convert the image pixel and actual size. The table used in this experiment is the Nittaku 3250 table. The white end line on this table measures $2 \mathrm{~cm}$ and conforms to the rules. The image width coordinate of the white end line (left and right) in Table 1 is $(X, Y)=(84,1097)$ and $(X, Y)=(124$ 1097). Thus, the white end line width is 40 pixels. We can then calculate that at the actual shooting site each pixel $2 \mathrm{~cm} / 40$ pixels $=0.05 \mathrm{~cm}$. Because the server is already serving on the side, Type 1 algorithm is used to automatically determine the ball location. The track is recorded, as shown in Figure 6. Figure 7 shows that pixel difference of the ball center position from when the ball leaves the hand to the apex of the toss is $113-75=38$ pixel. Thus, the ball height is 38 pixels $\times 0.05 \mathrm{~cm}=1.9 \mathrm{~cm}$. We can then judge that this service ball toss violated the rule. In addition, Type 2 algorithm can be used to automatically detect the hand's upper edge position, which can be used as a second verification for determining the position when the ball leaves the hand. 


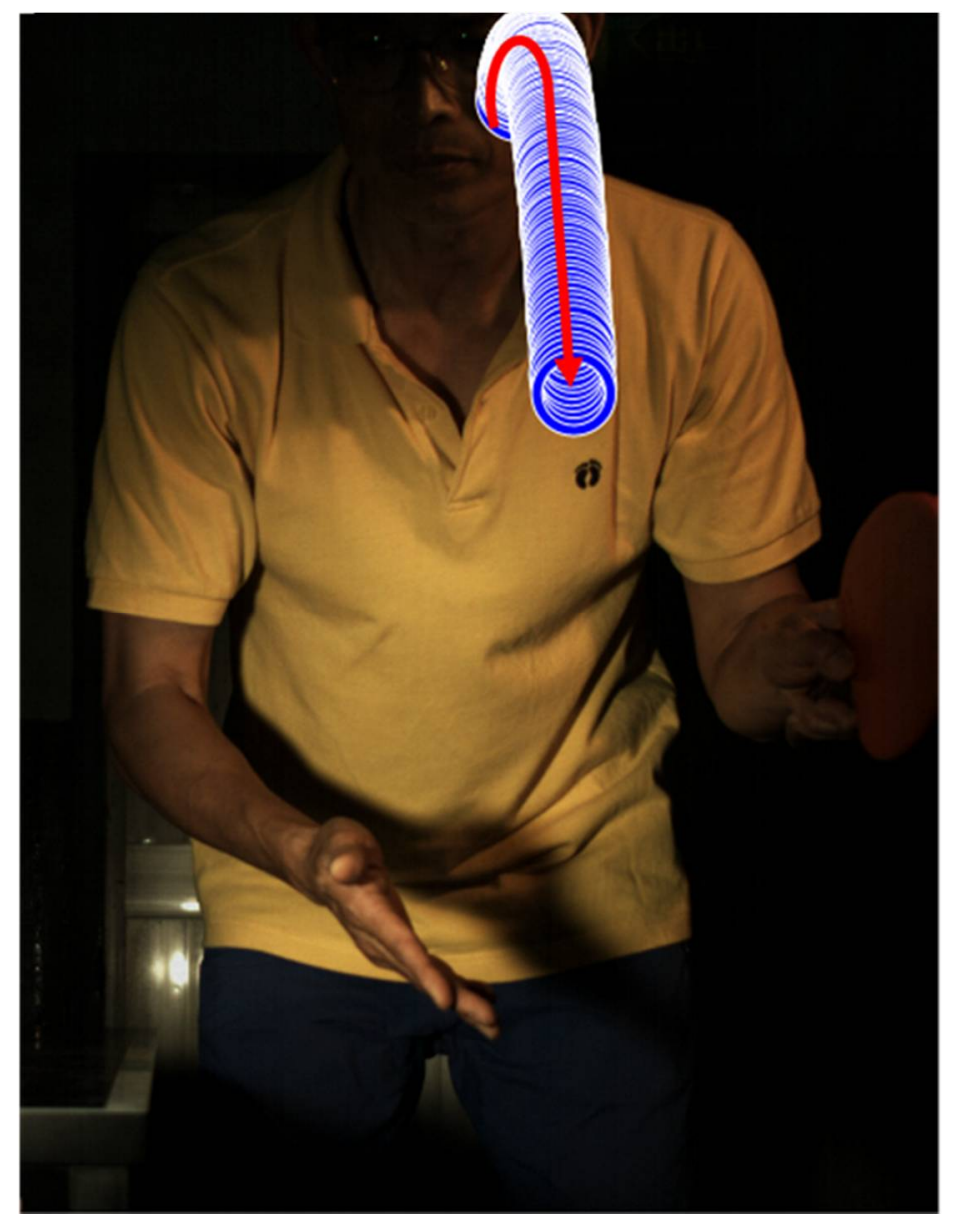

Figure 6. Automatic ball tracking and recording (recording starts the moment that the ball appears)
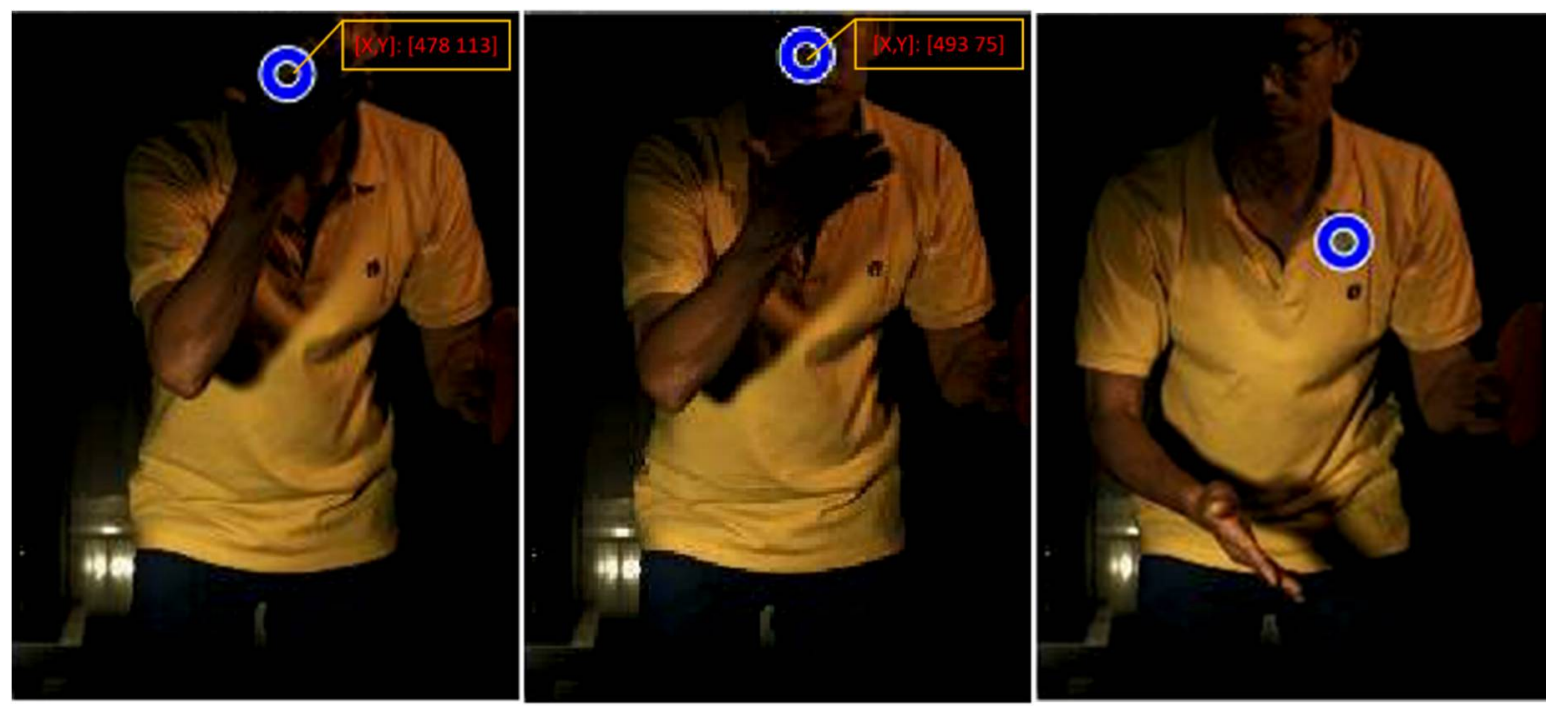

Figure 7. Ball position the moment it leaves the hand (left), the apex of the ball toss (middle), and the final frame position (right) 
Table 1. Proportion of actual size to pixel size

\begin{tabular}{|c|c|c|c|}
\hline & $\begin{array}{l}\text { Actual } \\
\text { width }\end{array}$ & $\begin{array}{c}\text { Image pixel } \\
\text { length }\end{array}$ & Position in the image \\
\hline & & & $\begin{array}{l}{[\mathrm{X}, \mathrm{Y}]:\left[\begin{array}{ll}84 & 1097\end{array}\right]} \\
{[\mathrm{R}, \mathrm{G}, \mathrm{B}]:\left[\begin{array}{lll}53 & 52 & 38\end{array}\right]}\end{array}$ \\
\hline $\begin{array}{l}\text { White end } \\
\text { line }\end{array}$ & $2 \mathrm{~cm}$ & 40 pixels & 5 미 \\
\hline
\end{tabular}

\section{Result and Discussion}

The height of the serve ball in table tennis must be higher than $16 \mathrm{~cm}$. However, without a clear image reference, accurately judging this with the naked eye is difficult. It only takes a few seconds to complete the table tennis service process (from serving preparation to service completion). Therefore, it is a challenge for image auxiliary to provide a judgment within short time after a service is completed. Image auxiliary must be able to rapidly identify the position where the ball leaves the hand and track the ball's movement, and then calculate the ball toss within the shortest time to provide image auxiliary judgment. The advantage of high-speed camera shooting is that each key frame can be obtained. The more frames taken each second the clearer the athlete's action and the track of the ball can be re-rendered. Although high-speed camera has this advantage, the short exposure time in high-speed photography requires strong lighting so that the light-sensitive components can successfully capture enough light. This is why additional strong light source is required. In this study we shot videos under simple light source conditions and used image processing techniques to automatically track ball and skin on the hand. We constructed a series of image processing methods to solve the low light source problem so that we do not affect the athlete. Different than Wong, who used Artificial Neural Network (ANN) technology to detect the circular table tennis [1], we implemented image segmentation on the color space to track the ball and the skin of the hand (results shown in Figure 2 - Figure 5). Hough transform can automatically and accurately find the ball position in a high noise thresholded image. The blue track in Figure 6 clearly shows the ball track. In the track display, the ball track conforms to the ball position in each frame. Thus, search of circular objects with Hough transform can resist noise. The result of this example is excellent. Once the ball position can be determined, we can also determine the point the ball leaves the hand and calculate the apex height of the ball toss. Ball track in Figure 6 shows that the height of the ball has not reached the regulated height, but the distance of the ball drop is very long. This can easily cause the umpire to misjudge and cause wrong cognition by the athlete. The primary objective of this study is to help the umpire determine whether a violation has occurred. Thus, the recorded video can be repeatedly replayed and the processed image can be superimposed in the video and displayed. This can help the umpire make the final judgment. During image processing, the pixel position that corresponds to the actual distance can be obtained from the target's actual size in proportion to the size of the pixels in the image. No complex calculation is required for the conversion. In consideration of computer calculation quantity and the possession of only one camera, we did not set up a mechanism to determine ball toss angle. We hope that future technological advances can solve problems posed by large calculation requirements and high-cost hardware so that studies in this field can make further breakthrough. 


\section{Conclusion}

In this study we used the characteristics of high-speed cameras to design an automatic image processing procedure, which can accurately record the key moment when the service ball leaves the hand during the toss and the apex of the ball toss. This can help umpires easily determine if a rule violation has occurred during the serve. The special feature of this study is using the actual size of the background object to calculate the actual distance that corresponds to the pixel size in the film. Thus, no complex calculation is required. In addition, image processing can be implemented in a low light source environment. Usually, with high-speed camera recording, light source become an important issue. However, we only used basic lighting in this study, and avoided interference from strong light sources. The algorithm was still able to automatically process the image within a short period of time.

Funding: This research received no external funding.

Acknowledgments: I would like to acknowledge Dr. Bo-Lin Jian's help to set up the experimental devices on this study.

Conflicts of Interest: The author declares no conflict of interest

\section{References}

1. Wong, P. K. C. Developing an intelligent assistant for table tennis umpires. In First Asia International Conference on Modelling and Simulation. Phuket, Thailand, 27-30 March, 2008.

2. "The International Table Tennis Federation Handbook 2017" https://d3mjm6zw6cr45s.cloudfront.net/2016/12/2017_ITTF_Handbook.pdf, accessed on 30/06/2018.

3. Wong, P. K. C. Developing an Intelligent Table Tennis Umpiring System: Identifying the ball from the scene. In Second Asia International Conference on Modelling \& Simulation. Kuala Lumpur, Malaysia, 13-15 May, 2008.

4. Myint, H.; Wong, P.; Dooley, L.; Hopgood, A. Tracking a table tennis ball for umpiring purposes. 2015 14th IAPR International Conference on Machine Vision Applications (MVA). Institute of Electrical and Electronics Engineers Inc. 2015, 170-173.

5. Desai, U. B. Merchant, S. N.; Zaveri Mukesh; Ajishna, G.; Purohit, M.; Phanish, H. S. Small object detection and tracking: Algorithm, analysis and application. Lecture Notes in Computer Science, Springer, Berlin 2005, ISSN 0302-9743.

6. Wong, K. C. P.; Dooley, L. S. High-motion table tennisball tracking for umpiring applications. In 2010 IEEE 10th International Conference on Signal Processing (ICSP). 2010, 2460-2463.

7. Yuen, H.; Princen, J.; Illingworth, J.; Kittler, J. Comparative study of Hough Transform methods for circle finding. Image Vis. Comput. 1990, 8, 71-77.

8. Davies, E. R. Computer and machine vision: theory, algorithms, practicalities, 4th ed. Amsterdam; Boston: Elsevier, 2012.

9. Atherton T. J.; Kerbyson, D. J. Size invariant circle detection. Image Vis. Comput. 1999, 17, 795-803.

10. Dong, P. Implementation of mathematical morphological operations for spatial data processing. Comput. Geosci. 1997, 23, 103-107.

11. Coupier, D.; Desolneux, A.; Ycart, B. Image Denoising by Statistical Area Thresholding. J. Math. Imaging Vis. 2005, 22, 183-197.

12. Kakumanu, P.; Makrogiannis, S.; Bourbakis, N. A survey of skin-color modeling and detection methods. Pattern Recognit. 2007, 40, 1106-1122.

13. Phung, S. L.; Bouzerdoum, A. Chai, D. Skin segmentation using color pixel classification: analysis and comparison. IEEE Trans. Pattern Anal. Mach. Intell. Jan, 2005, 27, 148-154.

14. Chaves-González, J. M.; Vega-Rodríguez, M. A.; Gómez-Pulido, J. A.; Sánchez-Pérez, J. M. Detecting skin in face recognition systems: A colour spaces study. Digit. Signal Process. 2010, 20, 806-823.

15. Cho, K.M.; Jang, J.H.; Hong, K.S. Adaptive skin-color filter. Pattern Recognit. 2001, 34, 1067-1073.

16. Sigal, L.; Sclaroff, S.; Athitsos, V. Skin color-based video segmentation under time-varying illumination. IEEE Trans. Pattern Anal. Mach. Intell. Jul, 2004, 26, 862-877. 
265

266

267

268

269

270
17. Q. Zhu, K.-T. Cheng, C.-T. Wu, Y.-L. Wu, Adaptive learning of an accurate skin-color model, in: Sixth IEEE International Conference on Automatic Face and Gesture Recognition, 2004. Proceedings., 2004: pp. 37-42. doi:10.1109/AFGR.2004.1301506.

18. E. Bayro-Corrochano, Geometric Algebra Tensor Voting, Hough Transform, Voting and Perception Using Conformal Geometric Algebra, in: Geometric Algebra Applications Vol. I, Springer, Cham, 2018: pp. 559611. doi:10.1007/978-3-319-74830-6 16. 